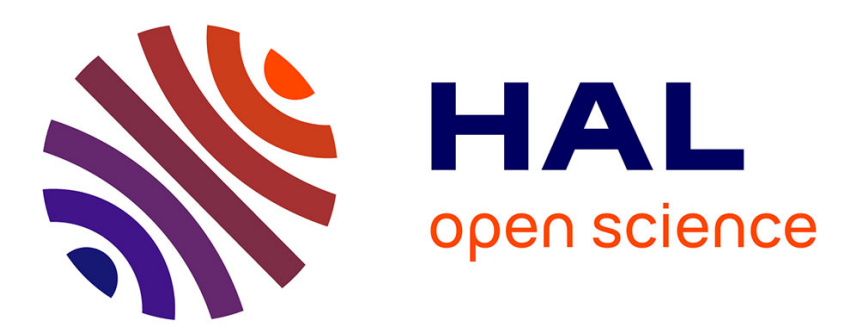

\title{
On dynamical feedback control design for generalized homogeneous differential inclusions
}

Konstantin Zimenko, Andrey Polyakov, Denis Efimov

\section{To cite this version:}

Konstantin Zimenko, Andrey Polyakov, Denis Efimov. On dynamical feedback control design for generalized homogeneous differential inclusions. CDC 2018 - 57th IEEE Conference on Decision and Control, Dec 2018, Miami Beach, United States. hal-01872548

\section{HAL Id: hal-01872548 \\ https://hal.inria.fr/hal-01872548}

Submitted on 12 Sep 2018

HAL is a multi-disciplinary open access archive for the deposit and dissemination of scientific research documents, whether they are published or not. The documents may come from teaching and research institutions in France or abroad, or from public or private research centers.
L'archive ouverte pluridisciplinaire HAL, est destinée au dépôt et à la diffusion de documents scientifiques de niveau recherche, publiés ou non, émanant des établissements d'enseignement et de recherche français ou étrangers, des laboratoires publics ou privés. 


\title{
On dynamical feedback control design for generalized homogeneous differential inclusions
}

\author{
Konstantin Zimenko, Andrey Polyakov and Denis Efimov*
}

\begin{abstract}
In the present paper, the stability criterion for generalized homogeneous differential inclusions is obtained that may simplify the control design procedure in some particular cases. Robust dynamical feedback control law is designed for homogeneous differential inclusions. Performance of the resulting dynamical feedback is illustrated by numerical simulation.
\end{abstract}

\section{INTRODUCTION}

Homogeneity is a property, that an object remains consistent with respect to a group of transformations. This property is a widely used tool for treatment of nonlinear systems: the homogeneity property can be used for stability/stabilization [1], [2], [3], [4], [5], [6], [7], output control design [16] and observer design [8], [9], [10], [11]. For example, the homogeneity property implies that in the case of negative homogeneity degree a locally asymptotically stable system is globally finitetime stable. In this paper the generalized homogeneity is utilized, which deals with the groups of linear transformations.

Extensions of homogeneity concept to differential inclusions (DI) have been proposed for different types of homogeneity in [21], [13], [14], [12]. DIs are related to many practical situations. For example, DIs appear

\footnotetext{
${ }^{*}$ The results on stabilization via dynamical feedback presented in Section 5 were developed under support of RSF (grant 17-19-01422) in ITMO University. The other research were partially supported by ANR 15 CE23 0007 (Project Finite4SoS) and the Ministry of Education and Science of Russian Federation (Projects 8.8885.2017/8.9, 14.Z50.31.0031)

$\dagger$ Konstantin Zimenko, Andrey Polyakov and Denis Efimov are with Department of Control Systems and Informatics, ITMO University, 49 Kronverkskiy av., 197101 Saint Petersburg, Russia. (e-mail: kostyazimenko@gmail.com).

${ }^{\ddagger}$ Andrey Polyakov and Denis Efimov are with Inria, 40 avenue Halley, 59650 Villeneuve d'Ascq, France (e-mail: denis.efimoveinria.fr, andrey.polyakove inria.fr). They are also with CRIStAL (UMR-CNRS 9189), Ecole Centrale de Lille, BP 48, Cite Scientifique, 59651 Villeneuve-d'Ascq, France.
}

handling with ordinary differential equations (ODE) with discontinuous right hand side (e.g., systems with switching devices, mechanical systems with dry friction, etc.) via regularization procedure [12]. Moreover, in some cases the transformation of nonlinear continuous ODE to the form of homogeneous DI may be more useful for stability analysis or control design. The procedure of representation of a nonlinear ODE in the form of homogeneous DI is called homogeneous extension.

This paper extends the results presented in [19] to homogeneous DIs. In particular, it is shown that asymptotically stable generalized homogeneous DI is equivalent to quadratically stable one via corresponding coordinate transformation. The coordinate transformations are based on utilizing the canonical homogeneous norm [15]. These results may significantly simplify stability analysis and control design procedures in some cases. Further, obtained results are used for dynamical feedback control design for homogeneous DIs. The control design is based on the scheme of a universal stabilizing control presented in [22]. Finally, numerical example and conclusions are presented. The presented example is noteworthy in that a continuous nonlinear and nonhomogeneous system is considered. Despite the continuity of ODE, it is shown that using homogeneous extension to represent the system as homogeneous DI, the synthesis of the finite-time control can be reduced to solving the system of linear matrix inequalities (LMI).

\section{NOTATION}

Through the paper the following notation will be used:

- $\mathbb{R}_{+}=\{x \in \mathbb{R}: x>0\}$, where $\mathbb{R}$ is the field of real numbers;

- $\|\cdot\|$ denotes a norm in $\mathbb{R}^{n}$;

- $\|A\|_{\mathbb{A}}=\sup _{x \in \mathbb{R}^{n}} \frac{\|A x\|}{\|x\|}$ for $A \in \mathbb{R}^{n \times n}$;

- $S=\left\{x \in \mathbb{R}^{n}:\|x\|=1\right\}$ is the unit sphere in $\mathbb{R}^{n}$;

- $I_{n} \in \mathbb{R}^{n \times n}$ is the identity matrix; 
- $\mathscr{C}^{n}(X, Y)$ is the set of continuously differentiable (at least up to the order $n$ ) maps $X \rightarrow Y$, where $X$ and $Y$ are open subsets of finite-dimensional spaces, $\mathscr{C}^{n}(X)=\mathscr{C}^{n}(X, \mathbb{R})$;

- the inequality $P>0$ means that the symmetric matrix $P=P^{T} \in \mathbb{R}^{n \times n}$ is positive definite;

- $P^{\frac{1}{2}}$ means that $P^{\frac{1}{2}}=M$ such that $P=M^{2}$;

- the minimal and maximal eigenvalues of a symmetric matrix $P=P^{T}$ are denoted by $\lambda_{\min }(P)$ and $\lambda_{\max }(P)$, respectively;

- $\mathfrak{R}(\lambda)$ denotes the real part of a complex number $\lambda$.

\section{PRELIMINARIES}

\subsection{Differential Inclusion}

The modern control theory frequently deals with systems modelled by ODEs with discontinuous righthand sides, which are accompanied by nonuniqueness of solutions. DIs are mainly introduced to capture behaviors of such systems. In this case standard assumptions of DI (it has non-empty, compact, convex values and it is upper semi-continuous) guarantee the existence of solutions [21].

Consider an ODE with discontinuous right-hand side

$$
\dot{x}=f(x), \quad f(0)=0, \quad x \in \mathbb{R}^{n},
$$

where $f$ is locally essentially bounded. This system can be replaced by DI using Filippov regularization procedure [12]:

$$
\dot{x} \in F(x)
$$

with set-valued map $F$ defined by

$$
F(x)=\bigcap_{\varepsilon>0} \bigcap_{N \in \mathscr{N}} \overline{\operatorname{conv}}(f(y), y \in B(x, \varepsilon) \backslash N),
$$

where $N \in \mathscr{N}$ means that the measure of $N$ is zero, $B(x, \varepsilon)$ is the ball with the center at $x \in \mathbb{R}^{n}$ and the radius $\varepsilon$.

Due to nonuniqueness of solutions, the system (2) may have some solutions, which converge to the origin while other solutions don't. In this case DIs may admit both weak and strong stability (see, for example, [12]). This paper deals only with the strong stability properties, which ask for stable behavior of all solutions of the system (2).

Note, that DIs can also be useful for systems with a continuous right-hand side. In some cases for stability analysis (control synthesis) of nonhomogeneous systems (1) it is more useful to represent the system in the form of homogeneous differential inclusion

$$
F(x)=\left\{e^{-v s} \mathbf{d}(-s) f(\mathbf{d}(s) x), \forall s \in \mathbb{R}\right\},
$$

called homogeneous extension [17].

\subsection{Generalized Homogeneity}

The homogeneity is a property that specifies sort of symmetry of an object with respect to a group of transformations (dilation operation). Generalized homogeneity (d-homogeneity) is a type of homogeneity, which deals with linear transformations (linear dilations).

Definition 1 [20], [15] A map $\mathbf{d}: \mathbb{R} \rightarrow \mathbb{R}^{n \times n}$ is called dilation in the space $\mathbb{R}^{n}$ if it satisfies:

- group property: $\mathbf{d}(0)=I_{n}$ and $\mathbf{d}(t+s)=$ $\mathbf{d}(t) \mathbf{d}(s)=\mathbf{d}(s) \mathbf{d}(t)$ for all $t, s \in \mathbb{R}$

- continuity property: $\mathbf{d}$ is a continuous map;

- limit property: $\quad \lim _{s \rightarrow-\infty}\|\mathbf{d}(s) x\|=0$ and $\lim _{s \rightarrow+\infty}\|\mathbf{d}(s) x\|=+\infty$ uniformly on the unit sphere $S$.

The dilation $\mathbf{d}$ is a uniformly continuous group [20]. The matrix $G_{\mathbf{d}} \in \mathbb{R}^{n \times n}$ defined by

$$
G_{\mathbf{d}}=\lim _{s \rightarrow 0} \frac{\mathbf{d}(s)-I_{n}}{s} .
$$

is known as the generator of the group $\mathbf{d}$ (see, for example, [18]). The generator $G_{\mathbf{d}}$ satisfies the following properties [18]:

$$
\begin{gathered}
\frac{d}{d s} \mathbf{d}(s)=G_{\mathbf{d}} \mathbf{d}(s)=\mathbf{d}(s) G_{\mathbf{d}} \\
\mathbf{d}(s)=e^{G_{\mathbf{d}} s}=\sum_{i=0}^{+\infty} \frac{s^{i} G_{\mathbf{d}}^{i}}{i !}
\end{gathered}
$$

where $s \in \mathbb{R}$.

Let us introduce the definitions on monotonicity of the dilation $\mathbf{d}(s)$.

Definition 2 [20] The dilation $\mathbf{d}$ is said to be monotone if $\|\mathbf{d}(s)\|_{\mathbb{A}}<1$ for all $s<0$.

Thus, monotonicity means that $\mathbf{d}(s)$ is strong contraction for $s<0$ (strong expansion for $s>0$ ) and implies that for any $x \in \mathbb{R} \backslash\{0\}$ there exists a unique pair $\left(s_{0}, x_{0}\right) \in \mathbb{R} \times S$ such that $x=\mathbf{d}\left(s_{0}\right) x_{0}$. The rate of contraction is introduced by the next definition.

Definition 3 [15], [19] The dilation $\mathbf{d}$ is said to be strictly monotone if $\exists \beta \in \mathbb{R}_{+}$such that $\|\mathbf{d}(s)\|_{\mathbb{A}} \leq e^{\beta s}$ for all $s \leq 0$. [15].

Monotonicity property may depend on a norm $\|\cdot\|$

Theorem 1 [19] If $\mathbf{d}$ is a dilation in $\mathbb{R}^{n}$, then 
- the generator matrix $G_{\mathbf{d}}$ is anti-Hurwitz, i.e. $\mathfrak{R}\left(\lambda_{i}\right)>0, i=1, \ldots, n$;

- there exists a matrix $P \in \mathbb{R}^{n \times n}$ such that

$$
P G_{\mathbf{d}}+G_{\mathbf{d}}^{T} P>0, \quad P>0 .
$$

- the dilation $\mathbf{d}$ is strictly monotone with respect to the weighted Euclidean norm $\|x\|=\sqrt{x^{T} P x}$ for $x \in$ $\mathbb{R}^{n}$ and P satisfying (7):

$$
\begin{aligned}
& e^{\alpha s} \leq\|\mathbf{d}(s)\|_{\mathbb{A}} \leq e^{\beta s} \quad \text { if } \quad s \leq 0, \\
& e^{\beta s} \leq\|\mathbf{d}(s)\|_{\mathbb{A}} \leq e^{\alpha s} \quad \text { if } \quad s \geq 0,
\end{aligned}
$$

where $\alpha=\frac{1}{2} \lambda_{\max }\left(P^{\frac{1}{2}} G_{\mathbf{d}} P^{-\frac{1}{2}}+P^{-\frac{1}{2}} G_{\mathbf{d}}^{T} P^{\frac{1}{2}}\right), \beta=$ $\frac{1}{2} \lambda_{\min }\left(P^{\frac{1}{2}} G_{\mathbf{d}} P^{-\frac{1}{2}}+P^{-\frac{1}{2}} G_{\mathbf{d}}^{T} P^{\frac{1}{2}}\right)$.

Definition 4 [20] A vector field $f: \mathbb{R}^{n} \rightarrow \mathbb{R}^{n}$ (a function $h: \mathbb{R}^{n} \rightarrow \mathbb{R}$ ) is said to be $\mathbf{d}$-homogeneous of degree $\mu \in \mathbb{R}$ if

$$
f(\mathbf{d}(s) x)=e^{\mu s} \mathbf{d}(s) f(x), \quad \forall x \in \mathbb{R}^{n} \backslash\{0\}, \quad \forall s \in \mathbb{R} .
$$$$
\text { (resp. } h(\mathbf{d}(s) x)=e^{\mu s} h(x), \forall x \in \mathbb{R}^{n} \backslash\{0\}, \quad \forall s \in \mathbb{R} \text {.) }
$$

Definition 5 A set-valued map $F: \mathbb{R}^{n} \rightrightarrows \mathbb{R}^{n}$ is said to be $\mathbf{d}$-homogeneous of degree $m \in \mathbb{R}$ if for all $x \in \mathbb{R}^{n}$ we have

$$
F(\mathbf{d}(s) x)=e^{m s} \mathbf{d}(s) F(x), \quad \forall x \in \mathbb{R}^{n} \backslash\{0\}, \quad \forall s \in \mathbb{R} .
$$

The system (2) is $\mathbf{d}$-homogeneous of degree $m$ if the set-valued map $F$ is homogeneous of degree $m$.

A special case of homogeneous function is a homogeneous norm [6], [19]: a continuous positive definite d-homogeneous function of degree 1 . Define the canonical homogeneous norm $\|\cdot\|_{\mathbf{d}}: \mathbb{R}^{n} \rightarrow \mathbb{R}_{+}$as $\|x\|_{\mathbf{d}}=e^{s_{x}}$, where $s_{x} \in \mathbb{R}$ is such that $\left\|\mathbf{d}\left(-s_{x}\right) x\right\|=1$. Note that $\|\mathbf{d}(s) x\|_{\mathbf{d}}=e^{s}\|x\|_{\mathbf{d}}$ and

$$
\left\|\mathbf{d}\left(-\ln \|x\|_{\mathbf{d}}\right) x\right\|=1 .
$$

If $\mathbf{d}$ is a strictly monotone dilation and the norm $\|\cdot\|$ is smooth outside the origin then homogeneous norm $\|\cdot\|_{\mathbf{d}}$ is also smooth outside the origin, $\frac{\partial\|\mathbf{d}(-s) x\|}{\partial s}<0$ if $s \in \mathbb{R}$, $x \in \mathbb{R}^{n} \backslash\{0\}$ and [19]

$$
\frac{\partial\|x\|_{\mathbf{d}}}{\partial x}=\left.\frac{\left.\|x\|_{\mathbf{d}} \frac{\partial\|z\|}{\partial z}\right|_{z=\mathbf{d}(-s) x}}{\left.\frac{\partial\|z\|}{\partial z}\right|_{z=\mathbf{d}(-s) x} G_{\mathbf{d}} \mathbf{d}(-s) x}\right|_{s=\ln \|x\|_{\mathbf{d}}} .
$$

Let us introduce the result on finite-time stability for $\mathbf{d}$-homogeneous set-valued maps.
Corollary 1 [21] Let $F$ be a $\mathbf{d}$-homogeneous setvalued map with degree $m<0$, satisfying the standard assumptions. Assume also that $F$ is strongly globally asymptotically stable. Then $F$ is strongly globally finitetime stable and the settling-time function is continuous at zero and locally bounded.

\section{QUADRATIC STABILITY OF HOMO- GENEOUS DIFFERENTIAL INCLU- SIONS}

Consider the system

$$
\dot{x} \in F(x), \quad x(0)=x_{0} \in \mathbb{R}^{n},
$$

where $F$ is a set-valued map satisfying the standard assumptions.

The next theorem shows that asymptotically stable DI (12) is topologically equivalent to quadratically stable one via corresponding coordinate transformation.

Theorem 2 Let $F$ be a $\mathbf{d}$-homogeneous set-valued map with degree $m$, satisfying the standard assumptions. Then the next claims are equivalent:

1. The system (12) is strongly globally asymptotically stable.

2. For all $k>\max (-m, 0)$, there exist a pair $(V, W)$ of continious functions, such that: $V \in \mathscr{C}^{\infty}\left(\mathbb{R}^{n}\right)$ is positive definite and homogeneous with degree $k ; W \in \mathscr{C}^{\infty}\left(\mathbb{R}^{n} \backslash\{0\}\right)$ is strictly positive outside the origin and homogeneous with degree $k+m$; $\max _{v \in F(x)} D V(x) v \leq-W(x)$ for all $x \neq 0$.

3. The origin of the system

$$
\dot{z} \in\|z\|^{1+m}\left(\frac{\left(I_{n}-G_{\mathbf{d}}\right) z z^{T} P}{z^{T} P G_{\mathbf{d}} z}+I_{n}\right) F\left(\frac{z}{\|z\|}\right),
$$

is asymptotically stable, where $\|z\|=\sqrt{z^{T} P z}$ with $P$ satisfying (7).

4. For any matrix $P \in \mathbb{R}^{n \times n}$ satisfying (7) there exists a d-homogeneous $\Psi \in \mathscr{C}^{\infty}\left(\mathbb{R}^{n} \backslash\{0\}\right)$ with degree 0 such that $\Psi$ is diffeomorphism on $\mathbb{R}^{n} \backslash\{0\}$, homeomorphism on $\mathbb{R}^{n}, \Psi(0)=0$ and

$$
\max _{\substack{v \in F(x) \\ x \in \mathbb{R}^{n}:\|\Psi(x)\|=1}} D\left(\Psi^{T}(x) P \Psi(x)\right) v<0
$$

Moreover, $\|\Psi\|_{\mathbf{d}} \in \mathscr{C}^{\infty}\left(\mathbb{R}^{n} \backslash\{0\}\right)$ is a Lyapunov function to the system (12), where $\|\cdot\|_{\mathbf{d}}$ is the canonical homogeneous norm induced by $\|x\|=$ $\sqrt{x^{T} P x}$ 
5. For any matrix $P \in \mathbb{R}^{n \times n}$ satisfying (7) there exists a map $\Xi \in \mathscr{C}^{\infty}\left(\mathbb{R}^{n} \backslash\{0\}, \mathbb{R}^{n \times n}\right)$ such that $\operatorname{det}(\Xi(z)) \neq 0, \frac{\partial \Xi(z)}{\partial z_{i}} z=0, \Xi\left(e^{s} z\right)=\Xi(z)$ for $z=$ $\left(z_{1}, \ldots, z_{n}\right)^{T} \in \mathbb{R}^{n} \backslash\{0\}, s \in \mathbb{R}, i=1, \ldots, n$ and

$$
\max _{v \in F\left(\frac{z}{\sqrt{z^{T} P z}}\right)} z^{T} \Xi^{T}(z) P \Xi(z)\left(\frac{\left(I_{n}-G_{\mathbf{d}}\right) z z^{T} P}{z^{T} P G_{\mathbf{d}} z}+I_{n}\right) v<0 .
$$

Analogously to [19] the expression (15) gives a stability criterion for generalized homogeneous DIs. Choosing $\Xi=I_{n}$ we derive the simplified sufficient stability condition

$$
\max _{v \in F\left(\frac{z}{\sqrt{z^{T} P z}}\right)} z^{T} P v<0 .
$$

\section{STABILIZATION VIA DYNAMICAL FEEDBACK}

Consider the system

$$
\dot{\sigma} \in G(\sigma, u)+\omega(t, \sigma, u),
$$

where $t \in \mathbb{R}_{+}$is time variable, $\sigma(t) \in \mathbb{R}^{n}$ is the state vector, $u(t) \in \mathbb{R}^{l}$ is the control input, $G: \mathbb{R}^{n+l} \rightrightarrows \mathbb{R}^{n}$ is a set-valued map and the function $\omega: \mathbb{R}^{n+1} \rightarrow \mathbb{R}^{n}$ is assumed to be unknown.

Based on the scheme of a universal stabilizing control design given in [22] the following result can be derived utilizing Theorem 2 .

Theorem 3 (On homogeneous dynamical feedback) Let $\mathbf{d}_{x}$ and $\mathbf{d}_{u}$ be dilations in $\mathbb{R}^{n}$ and $\mathbb{R}^{l}$, respectively, $\mathbf{d}:=\left(\begin{array}{cc}\mathbf{d}_{x}(s) & 0 \\ 0 & \mathbf{d}_{u}(s)\end{array}\right)$ and $\tilde{F}=\left(\begin{array}{c}G \\ 0\end{array}\right): \mathbb{R}^{n+l} \rightrightarrows$ $\mathbb{R}^{n+l}$ is a d-homogeneous set-valued map with degree $\tilde{m}$. The origin of the system (16) with $\omega=0$ is globally asymptotically stabilizable by means of the homogeneous dynamical feedback

$$
\dot{u}(t)=k(\sigma(t), u(t)), \quad k \in \mathscr{C}\left(\mathbb{R}^{n+l} \backslash\{0\}, \mathbb{R}^{l}\right)
$$

with d-homogeneous $\left(\begin{array}{c}G \\ k\end{array}\right): \mathbb{R}^{n+l} \rightrightarrows \mathbb{R}^{n+l}$ if and only if there exist a number $\gamma \geq 0$, a symmetric matrix $P \in$ $\mathbb{R}^{(n+l) \times(n+l)}$ satisfying (7) and a map $\Xi \in \mathscr{C}^{\infty}\left(\mathbb{R}^{n+l} \backslash\right.$ $\left.\{0\}, \mathbb{R}^{(n+l) \times(n+l)}\right)$ :

$$
\begin{aligned}
& \operatorname{det}(\Xi(z)) \neq 0, \frac{\partial \Xi(z)}{\partial z_{i}} z=0, \Xi\left(e^{s} z\right)=\Xi(z) \text { for } \\
& z=\left(z_{1}, \ldots, z_{n+l}\right)^{T} \in \mathbb{R}^{n+l} \backslash\{0\}, s \in \mathbb{R}, i=1, \ldots, n+l
\end{aligned}
$$

such that

$$
a(z)<\gamma \sqrt{b^{T}(z) b(z)} \quad \text { for } \quad z \in S
$$

where

$$
\begin{aligned}
& a(z)=\max _{v \in \tilde{F}(z)} z^{T} W(z) v, \quad b^{T}(z)=z^{T} W(z)\left(\begin{array}{c}
0 \\
I_{l}
\end{array}\right), \\
& W(z)=\Xi^{T}(z) P \Xi(z)\left(\frac{\left(I_{n+l}-G_{\mathbf{d}}\right) z^{T} z P}{z^{T} P G_{\mathbf{d}}}+I_{n+l}\right)
\end{aligned}
$$

and $S$ is the unit sphere in $\mathbb{R}^{n+l}$ with $\|z\|=\sqrt{z^{T} P z}$. Moreover, the corresponding stabilizing homogeneous feedback law can be designed as follows

$k(\xi)=\|\xi\|_{\mathbf{d}}^{\tilde{m}} \mathbf{d}_{u}\left(\ln \|\xi\|_{\mathbf{d}}\right) k_{0}\left(\mathbf{d}\left(-\ln \|\xi\|_{\mathbf{d}}\right) \xi\right), \quad \xi=\left(\begin{array}{l}\sigma \\ u\end{array}\right)$,

where

$k_{0}(\cdot)=\left\{\begin{array}{cc}-\frac{a(\cdot)+\sqrt{a^{2}(\cdot)+\left(b^{T}(\cdot) b(\cdot)\right)^{2}}}{b^{T}(\cdot) b(\cdot)} b(\cdot) & \text { if } \quad b(\cdot) \neq 0 \\ 0 & \text { if } \quad b(\cdot)=0\end{array}\right.$

and $\|\cdot\|_{\mathbf{d}}$ is the canonical homogeneous norm induced by $\|\xi\|=\sqrt{\xi^{T} P \xi}$.

For $\Xi=I_{n+l}$ the inequality (18) gives the following sufficient condition of quadratic stabilizability:

$$
\begin{aligned}
& \max _{v \in \tilde{F}\left(\frac{z}{\sqrt{z^{T} P z}}\right)} z^{T} P v \leq \gamma \sqrt{z^{T} P\left(\begin{array}{ll}
0 & 0 \\
0 & I_{l}
\end{array}\right) P z, z \neq 0,} \\
& P G_{\mathbf{d}}+G_{\mathbf{d}}^{T} P>0, \quad P>0 .
\end{aligned}
$$

The following theorem presents the modification of the control scheme from Theorem 3 to reject some additive disturbances.

Theorem 4 (On robust homogeneous dynamical feedback) Let conditions of Theorem 3 hold and

$$
\begin{aligned}
& \max _{v \in F\left(\frac{z}{\|z\|}\right)} z^{T} \Xi^{T}(z) P \Xi(z)\left(\frac{\left(I_{n+l}-G_{\mathbf{d}}\right) z z^{T} P}{z^{T} P G_{\mathbf{d}} z}+I_{n+l}\right) \times \\
& \left(v+\frac{\mathbf{d}(-\ln \|z\|) \tilde{\omega}}{\|z\|^{\|\|^{m}}}\right)<0, \quad z \neq 0,
\end{aligned}
$$

where $z=\|\xi\|_{\mathbf{d}} \mathbf{d}\left(-\ln \|\xi\|_{\mathbf{d}}\right) \xi,\|z\|=\sqrt{z^{T} P z}, \quad \tilde{\omega}=$ $\left(\begin{array}{c}\omega \\ 0\end{array}\right), \xi=\left(\begin{array}{l}\sigma \\ u\end{array}\right) \in \mathbb{R}^{n+l}$. Then the closed-loop system (16), (17) is globally asymptotically stable.

More constructive (but also more conservative) sufficient condition can be derived under an assumption on quadratic stabilizability of the nominal system.

Corollary 2 Let conditions of Theorem 4 hold for $\Xi=I_{n+l}$ and

$$
\sqrt{\tilde{\omega}^{T} P \tilde{\omega}}<-\max _{v \in F\left(\frac{z}{\|z\|}\right)} z^{T} P v\left\{\begin{array}{lll}
\|z\|^{\alpha+\tilde{m}-1} & \text { if } & 0<\|z\| \leq 1, \\
\|z\|^{\beta+\tilde{m}-1} & \text { if } & \|z\|>1,
\end{array}\right.
$$

where $z=\|\xi\|_{\mathbf{d}} \mathbf{d}\left(-\ln \|\xi\|_{\mathbf{d}}\right) \xi,\|z\|=\sqrt{z^{T} P z}, \tilde{\omega}=$ $\left(\begin{array}{c}\omega \\ 0\end{array}\right), \xi=\left(\begin{array}{l}\sigma \\ u\end{array}\right) \in \mathbb{R}^{n+l}$ and $\alpha, \beta$ are defined in Theorem 1. Then the closed-loop system (16), (17) is globally asymptotically stable. 


\section{NUMERICAL EXAMPLE}

Consider the stabilization problem for the system

$$
\dot{\sigma}_{1}=\left|\sigma_{1}\right|^{\frac{1}{3}} u+\sigma_{2}\left(1+0.5 \sin \left(\sigma_{1}\right)\right), \quad \dot{\sigma}_{2}=u,
$$

where $\sigma_{1}, \sigma_{2}, u \in \mathbb{R}$. Using the homogeneous extension (4) for the system (22) obtain the set-valued map $\tilde{F}: \mathbb{R}^{3} \rightrightarrows \mathbb{R}^{3}$

$$
\tilde{F}(\xi)=\left(\begin{array}{c}
\left|\xi_{1}\right|^{\frac{1}{3}} \xi_{3}+(1+[-0.5,0.5]) \xi_{2} \\
\xi_{3} \\
0
\end{array}\right)
$$

where $\xi=\left(\xi_{1}, \xi_{2}, \xi_{3}\right)^{T}=\left(\sigma_{1}, \sigma_{2}, u\right)^{T}$. Note, that $\tilde{F}(\xi)$ is d-homogeneous of degree -1 with respect to the dilation $\mathbf{d}$ in $\mathbb{R}^{3}$ generated by $G_{\mathbf{d}}=\operatorname{diag}\{3,2,1\}$. Hence, the sufficient stabilizability condition (18) becomes

$$
\begin{gathered}
z^{T} P A(z) z<\gamma \sqrt{z^{T} P z} \sqrt{z^{T} P e_{3} e_{3}^{T} P z}, \\
P>0, \quad P G_{\mathbf{d}}+G_{\mathbf{d}}^{T} P>0,
\end{gathered}
$$

where $z=\left(z_{1}, z_{2}, z_{3}\right)^{T} \in \mathbb{R}^{3}, e_{3}=(0,0,1)^{T} \in \mathbb{R}^{3}$ and

$$
A(z)=\left(\begin{array}{ccc}
0 & {[0.5,1.5]} & \left(\frac{\left|z_{1}\right|}{\sqrt{z^{T} P z}}\right)^{\frac{1}{3}} \\
0 & 0 & 1 \\
0 & 0 & 0
\end{array}\right) .
$$

Let $X \in \mathbb{R}^{3 \times 3}$ and $\gamma_{0}>0$ satisfy the linear matrix inequalities

$X A_{i}^{T}+A_{i} X-2 \gamma_{0} e_{3} e_{3}^{T}<0, X G_{\mathbf{d}}+G_{\mathbf{d}} X>0, X>0, i=\overline{1,4}$

for $A_{1}=\left(\begin{array}{ccc}0 & 0.5 & 0 \\ 0 & 0 & 1 \\ 0 & 0 & 0\end{array}\right), \quad A_{2}=\left(\begin{array}{ccc}0 & 1.5 & 0 \\ 0 & 0 & 1 \\ 0 & 0 & 0\end{array}\right)$, $A_{3}=\left(\begin{array}{ccc}0 & 0.5 & 1 \\ 0 & 0 & 1 \\ 0 & 0 & 0\end{array}\right), \quad A_{4}=\left(\begin{array}{ccc}0 & 1.5 & 1 \\ 0 & 0 & 1 \\ 0 & 0 & 0\end{array}\right)$, $e_{3}=\left(\begin{array}{lll}0 & 0 & 1\end{array}\right)^{T}$. Choose $P=\tilde{P} / \tilde{p}_{11}, \quad \tilde{P}=X^{-1}$ that $\left(\frac{\left|z_{1}\right|}{\sqrt{z^{T} P z}}\right)^{\frac{1}{3}} \in[0,1]$. Then, since $A(z)$ can be represented as $A(z)=\sum_{i=1}^{4} \alpha_{i} A_{i}$ for $\alpha_{i} \in[0,1]: \sum_{i} \alpha_{i}=1$, the stability condition (18) holds.

Finally, the control law (19) takes the form

$$
\dot{u}=k_{0}\left(\mathbf{d}\left(-\ln \|\xi\|_{\mathbf{d}}\right) \xi\right),
$$

for $a(z)=\max \frac{z^{T} P A(z) z}{\sqrt{z^{T} P z}}, b(z)=p_{13} z_{1}+p_{23} z_{2}+p_{33} z_{3}$ and stabilizes the system (22) in finite time (due to negative degree of homogeneity).
The numerical simulation with $\sigma_{0}=\left(\begin{array}{ll}1 & 0\end{array}\right)^{T}$ and the matrix

$$
P=\left(\begin{array}{lll}
1.0000 & 1.2870 & 0.9848 \\
1.2870 & 2.3398 & 1.7059 \\
0.9848 & 1.7059 & 1.5910
\end{array}\right)
$$

satisfying the above-mentioned LMIs is shown in Fig. 1, 2. The simulation of the closed-loop system has been done by the Euler method with the fixed step size $h=0.01$. The parameter $a(z)$ has been calculated on the numerical grid over the interval $[0.5,1.5]$ for $A_{12}(z)$ at each sampling step. Note, that $a(z)$ can also be calculated off-line on some grid (which is constructed in some operation domain), approximated and stored in the controller for on-line usage.

Note, that in the case of feedback synthesis based on the analogous result for ODE [19] it is necessary to consider the term $0.5 \sigma_{2} \sin \left(\sigma_{1}\right)$ in (22) as perturbation to meet additional condition. Compared with this, the use of homogeneous extension and Theorem 3 allowed to synthesize the control law with LMIs solving only.

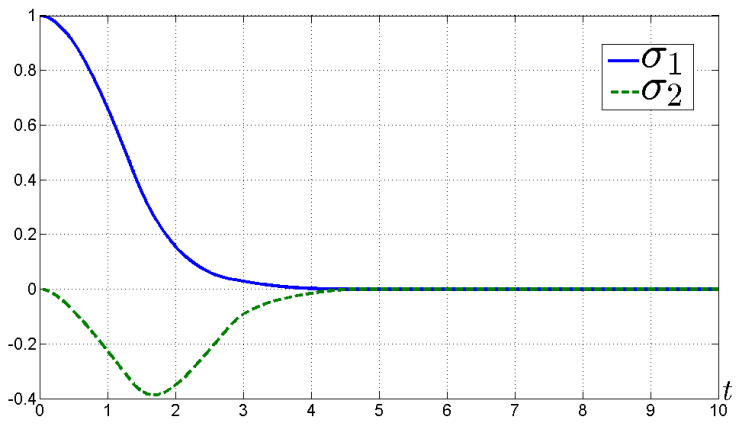

Figure 1. Evolution of the state of the closedloop system

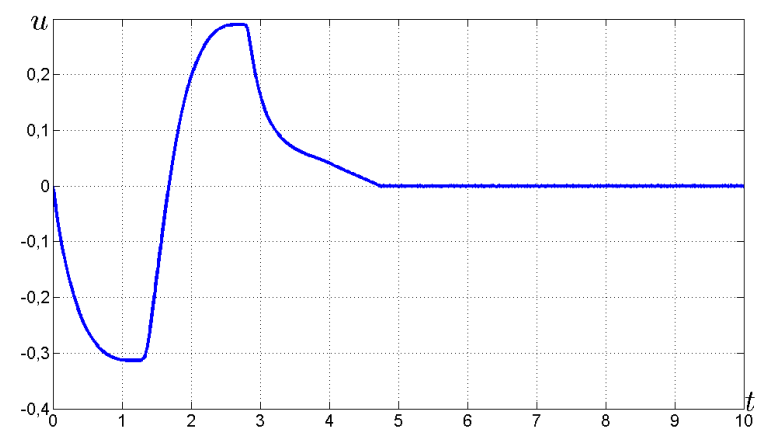

Figure 2. Evolution of the control signal 


\section{CONCLUSIONS}

The paper extends the results presented in [19] for generalized homogeneous differential inclusions. It has been shown that asymptotically stable DI is equivalent to quadratically stable one via corresponding coordinate transformation. The stability criterion is given that may simplify the control design procedure in some cases. This results were used for feedback dynamical control design. In the numerical example the homogeneous extension is used to represent continuous system in the form of generalized homogeneous DI. For obtained DI the synthesis of the algorithm for parameter tuning of finite-time dynamical feedback is presented in terms of LMIs.

\section{References}

[1] E. Moulay, W. Perruquetti, Finite-time stability and stabilization: State of the art, Lecture Notes in Control and Information Sciences, vol. 334, 2006, pp. 23-41.

[2] V.I. Zubov, On systems of ordinary differential equations with generalized homogeneous right-hand sides, Izvestia vuzov. Mathematica, vol. 1, 1958, pp. 80-88 (in Russian).

[3] V. I. Zubov, Methods of A.M. Lyapunov and Their Applications, Noordhoff, Leiden, 1964.

[4] A. Polyakov, D. Efimov, W. Perruquetti, Robust Stabilization of MIMO Systems in Finite/Fixed Time, Int. J. Robust. Nonlinear Control, vol. 26(1), 2016, pp. 69-90.

[5] A. Bacciotti, L. Rosier, Lyapunov Functions and Stability in Control Theory, Springer, 2005.

[6] M. Kawski, Geometric Homogeneity and Stabilization, in Proc. IFAC Nonlinear Control Symposium, A. Krener and D. Mayne, Eds., Lake Tahoe, CA, 1995, pp. 164169.

[7] H. Rios, D. Efimov, L. Fridman, J. Moreno, W. Perruquetti, Homogeneity Based Uniform Stability Analysis for Time-Varying Systems, IEEE Transactions on Automatic Control, vol. 61(3), 2016, pp. 725-734. )

[8] W. Perruquetti, T. Floquet, and E. Moulay, Finite-time observers: Application to secure communication,IEEE Transactions on Automatic Control, vol. 53(1), 2008, pp. 356-360.

[9] A. Levant, M. Livne, Exact Differentiation of Signals With Unbounded Higher Derivatives, IEEE Transactions on Automatic Control, vol. 57(4), 2012, pp. 10761080.

[10] T. Menard, E. Moulay, W. Perruquetti, A global highgain finite-time observer, IEEE Transactions on Automatic Control, vol. 55(6), 2010, pp. 1500-1506.

[11] Y. Shen, Y. Huang, J. Gu, Global finite-time observers for lipschitz nonlinear systems, IEEE Transactions on Automatic Control, vol. 56(2), 2011, pp. 418-424.

[12] A. Filippov, Differential equations with discontinuous right-hand sides, Dordrecht, Kluwer, 1988.
[13] A. Levant, Homogeneity approach to high-order slidingmode design, Automatica, vol. 41(5), 2005, pp. 823830.

[14] Y. Orlov, Finite Time Stability and Robust Control Synthesis of Uncertain Switched Systems, SIAM Journal on Control and Optimization, vol. 43(4), 2005, pp. 12531271.

[15] A. Polyakov, J.-M. Coron, L. Rosier, On finite-time stabilization of evolution equations: A homogeneous approach, in Conference on Decision and Control, 2016, pp. 3143-3148.

[16] V. Andrieu, L. Praly, A. Astolfi, Homogeneous Approximation, Recursive Observer Design, and Output Feedback, SIAM Journal on Control and Optimization, vol. 47(4), 2008, pp. 1814-1850.

[17] A. Polyakov, J.-M. Coron, L. Rosier, On Homogeneous Finite-Time Control for Linear Evolution Equation in Hilbert Space, IEEE Transactions on Automatic Control, 2018.

[18] A. Pazy, Semigroups of Linear Operators and Applications to Partial Differential Equations, Springer, 1983.

[19] A. Polyakov, Sliding Mode Control Design Using Canonical Homogeneous Norm, Int. J. Robust. Nonlinear Control, 2018, pp. 1-20.

[20] A. Polyakov, D. Efimov, E. Fridman, W. Perruquetti, On Homogeneous Distributed Parameter Systems, IEEE Transactions on Automatic Control, vol. 61(11), 2016, pp. 3657-3662.

[21] E. Bernuau, D. Efimov, W. Perruquetti, A. Polyakov, On an Extension of Homogeneity Notion for Differential Inclusions, European Control Conference (ECC), 2013, pp. 2204-2209.

[22] E. Sontag, A 'universal' construction of Artstein's theorem on nonlinear stabilization, Systems \& Control Letters, vol. 13, 1989, pp. 117-123. 